\title{
Unique Features and Properties of Nanostructured Materials
}

\author{
Horst Hahn
}

Technische Universität Darmstadt, Institute of Materials Science, Thin Films Division, Darmstadt, Germany

\section{Abstract}

In this introductory paper an attempt is made to give an overview of the area of nanostructured „materials irrespective of the synthesis process. The various microstructural features such as clusters or isolated nanoparticles, agglomerated nanopowders, consolidated nanomaterials and nanocomposite materials as well as all materials classes are considered. As an important component of modern research on nanomaterials a section describes the various characterization tools available. Based on these remarks some properties of nanostructured materials will be summarized emphasizing the property-microstructure relationships. Finally, a brief outlook on applications and initial industrial use of nanomaterials is presented.

\section{Introduction}

Nanostructures are plentiful in nature. In the universe nanoparticles are distributed widely and are considered to be the building blocks in planet formation processes. Biological systems have built up inorganic-organic nanocomposite structures to improve the mechanical properties or to improve the optical, magnetic and chemical sensing in living species. As an example, nacre (mother-of-pearl) from the mollusc shell is a biologically formed lamellar ceramic, which ,exhibits structural robustness despite the brittle nature of its constituents. [1] Figure 1 shows an SEM imge of a fracture surface of an abalone shell exhibiting the $\mathrm{CaCO}_{3}$-platelets which are se-

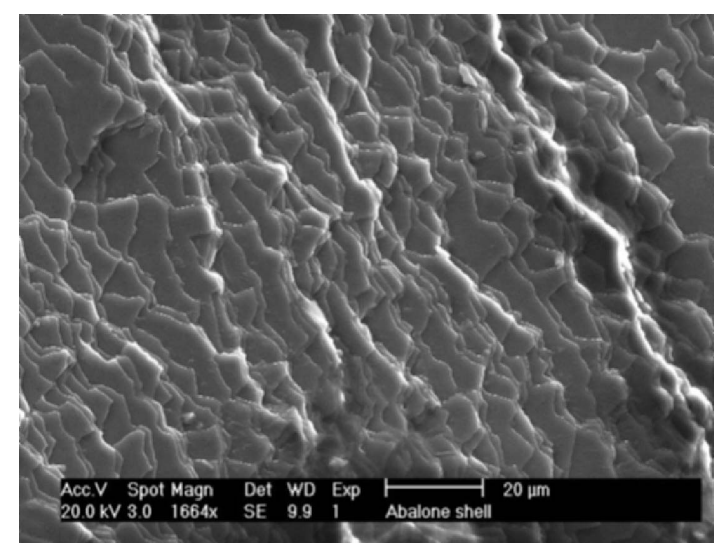

Figure 1: SEM image of a fracture surface of a Korean abalone shell showing the individual calcium-carbonate platelets separated by organic compounds.

Nanomaterials by Severe Plastic Deformation. Edited by M. J. Zehetbauer and R. Z. Valiev Copyright $\odot 2004$ WILEY-VCH Verlag GmbH \& Co. KGaA, Weinheim ISBN: 3-527-30659-5 
parated by organic compounds which exhibit nanometer dimensions. These systems have evolved and been optimized by evolution over millions of years into sophisticated and complex structures. In natural systems the bottom-up approach starting from molecules and involving self organization concepts has been highly successful in building larger structural and functional components. Functional systems are characterized by complex sensing, self repair, information transmission and storage and other functions all based on molecular building blocks. Examples of these complex structures for structural purposes are teeth, such as shark teeth, which consist of a composite of biomineralized fluorapatite and organic compounds. These structures result in the unique combination of hardness, fracture toughness and sharpness, see Figure 2.

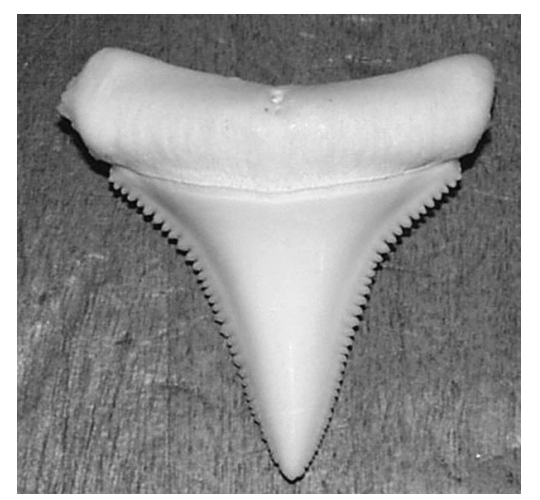

Figure 2: Example of a nanostructure found in nature: shark tooth with unique mechanical properties. The overall dimension of the tooth can reach several $\mathrm{cm}$.

Another example for a biological nanostructure is opal which exhibits unique optical properties. The self cleaning effects of the surfaces of the lotus flower have been attributed to the combined micro- and nanostructure which in combination with hydrophobic groups give the surface a water and dirt repellent behavior. [2] In the past few years, numerous companies have realized products resembling the surface morphology and chemistry of the lotus flower such as paint, glass surface and ceramic tiles with dirt repellent properties. The realization that nature can provide the model for improved engineering has created a research field called ,bio-,,mimicking or bio-inspired materials science. It has been possible to process these ceramic-organic nanocomposite structures which provide new technological opportunities and potential for applications. [3] Other exciting results have been published such as the biomimetic growth of synthetic fluorapatite [4] in the laboratory and promising new technical applications of these nanomaterials are envisioned. [5] Other man-made nanostructures were manufactured for their attractive optical properties, such as the colloidal gold particles in glass as seen in medieval church windows.

While plentiful man made materials with nanostructures have been in use for a long time (partially without knowing it) a change of the scientific and technological approach can be identified over the past two decades. This change can be related to a few key ideas and discoveries: the idea of assembling nanostructures from atomic, molecular or nanometer sized building blocks, [6] the discovery of new forms of carbon, i.e., fullerenes [7] and carbon nanotubes, and the development of scanning probe microscopy, [8] such as scanning tunneling microscopy (STM) and atomic force microscopy (AFM). With the visionary goals many researchers world- 
wide have worked intensively on the development of novel or improved synthesis methods, new and better characterization techniques and the measurement and the design of the properties of nanostructured materials. In this paper some aspects of the immensely wide field will be described. However, as the field of nanostructured materials is very broad including all classes of materials as well as composites it is not possible and not attempted to consider all developments and all research groups and industries working in this area.

\section{Synthesis}

The microstructure and properties of nanostructured materials depend in an extreme manner on the synthesis method as well as on the processing route. Therefore, it is of utmost importance to select the most appropriate technique for preparation of nanomaterials with desired properties and property combinations. Synthesis techniques can be divided into bottom-up and top-down approaches. The top-down approach starts with materials with conventional crystalline microstructures, typically metals and alloys, and defects such as dislocations and point defects are introduced by severe plastic deformation such as in equal channel pressing. The recrystallization of the material leads to finer and finer grain sizes and under certain processing conditions to nanostructured materials. The advantage of these approaches is the fact that bulk nanostructured materials with theoretical density can be prepared. An alternative to obtain theoretical dense materials is the pulsed electrodeposition method developed by Erb and El-Sherik which yields nanocrystalline strips, however, only with thicknesses of several hundred microns. [9] The bottom-up approach includes many different techniques which are based on liquid or gas phase processes. Classically, wet chemical processes such as precipitation and sol-gel have been employed to obtain nanoparticles, however, with the disadvantage of severe agglomeration. In the gas phase metallic and ceramic nanoparticles have been synthesized by using Inert Gas Condensation, Flame Pyrolysis (Aerosol process by Degussa) and chemical vapor based processes. The major microstructural features in preparing nanoparticles for subsequent use are: nanometer sized primary particles with narrow size distributions, minimum amount of agglomeration, good crystallinity, etc.

Two techniques, chemical vapor synthesis (CVS) in the gas phase [10] and electrodeposition under oxidizing conditions (EDOC) in the liquid phase, [11] together with the resulting microstructures will be presented in more detail and the advantages and disadvantages be discussed. CVS is based on chemical vapor deposition (CVD) for the synthesis of thin films and coatings by the decomposition of metalorganic precursors. Whether thin films are deposited by heterogeneous ,nucleation or nanoparticles are formed in the gas phase by homogeneous nucleation is determined by the residence time of the precursor in the hot zone of the reactor. The most important parameters determining the growth regime and the particle size are the total pressure, the precursor partial pressure and the temperature of the reaction zone. A typical reactor set-up is shown schematically in Figure 3 with one precursor source, the hot wall reactor, the thermophoretic collector, the pumping unit and the control devices for pressure and temperature. The hot wall concept operating at reduced pressures has been successfully scaled up in a cooperation project with a large German corporation involved in the synthesis of nanopowders such as carbon black, titania and silica. [12] 


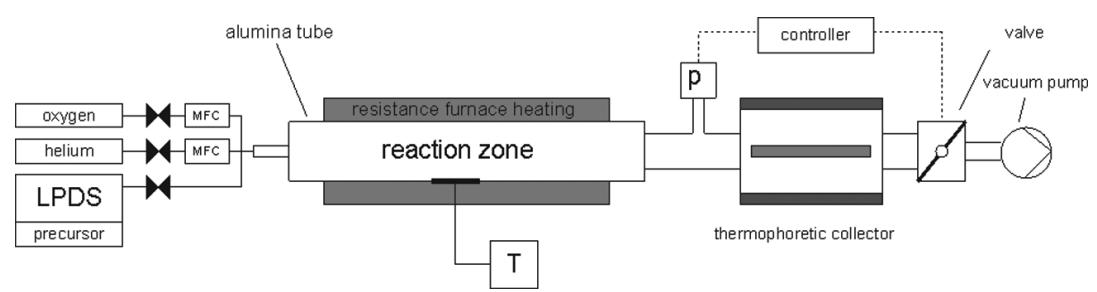

Figure 3: Schematic diagram of the major components of a CVS hot wall reactor: precursor source (liquid precursor delivery system, LPDS), hot wall reaction zone, thermophoretic particle collector, pumping system, and pressure and temperature control.

When two precursors are used, the precursor delivery can be modified in the following way:

(1) two precursors are introduced simultaneously into the reaction zone yielding doped nanoparticles (i.e. alumina doped zirconia); [13]

(2) two precursors are introduced into two concentric reaction tubes, reacted to form nanoparticles and then mixed in the gas phase to yield a nanocomposite structure (i.e., alumina mixed with zirconia) and

(3) in the first reaction zone the first precursor is decomposed to form nanoparticles by homogeneous nucleation which are subsequently coated in a second reaction zone by introducing the second precursor under conditions which favor CVD deposition (i.e., alumina surface coated zirconia). [14]

The experimental set-up of case 3) can be further modified by using a plasma reaction zone with pulse option which allows the controlled functionalization with organic molecules and polymeric shells. [15,16] Figure 4 shows a high resolution electron image of polymer coated titania nanoparticles where the crystalline titania core can be clearly distinguished from the amorphous organic shell on several grains.

Further evidence of the complete coating can be obtained by surface analysis, FTIR studies and by dispersion ,experiments in different organic liquids and water. The modification of the surfaces of nanoparticles allows the improvement of dispersibility in various aqueous and organic solvents which is important for many ceramic processing steps (dip- or spin-coating, slurries for ceramic processing, etc.) and for technical applications of dispersions. Additionally, the inorganic core/polymer shell structure allows the preparation of polymer nanocomposites with excellent separation between the inorganic nanoparticles.

A further variation by exact control of all synthesis parameters allows the growth of thick nanocrystalline coatings on dense and porous substrates. Depending on the substrate temperature the porosity of the coating can be changed over a wide range up to theoretical density. This intermediate stage, called CVD/CVS, has been successfully used to deposit a nanocrystalline coating of yttrium stabilized zirconia on porous anode substrates for high temperature solid oxide fuel cell applications. Figure 5 shows a high resolution scanning electron image of a coated anode substrate. [17]

The processes leading to particle formation have been modeled and simulated by many authors. The detailed description of these efforts is beyond the scope of this paper. A comprehen- 


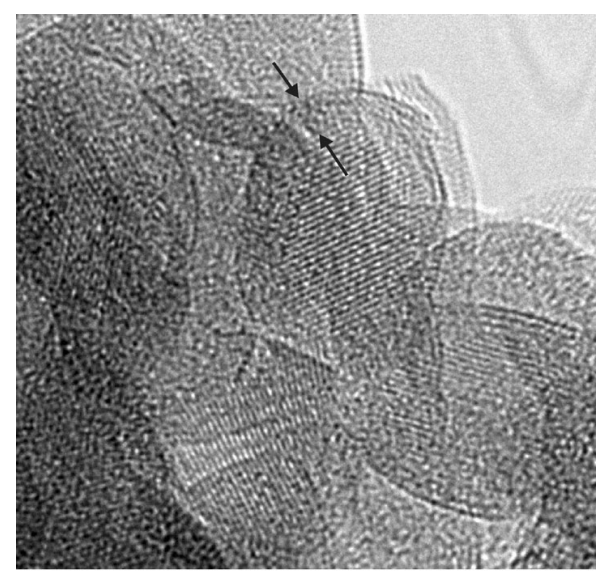

$5 \mathrm{~nm}$

Figure 4: High resolution TEM of titania nanoparticles (crystalline core) coated with an amorphous polymeric shell.

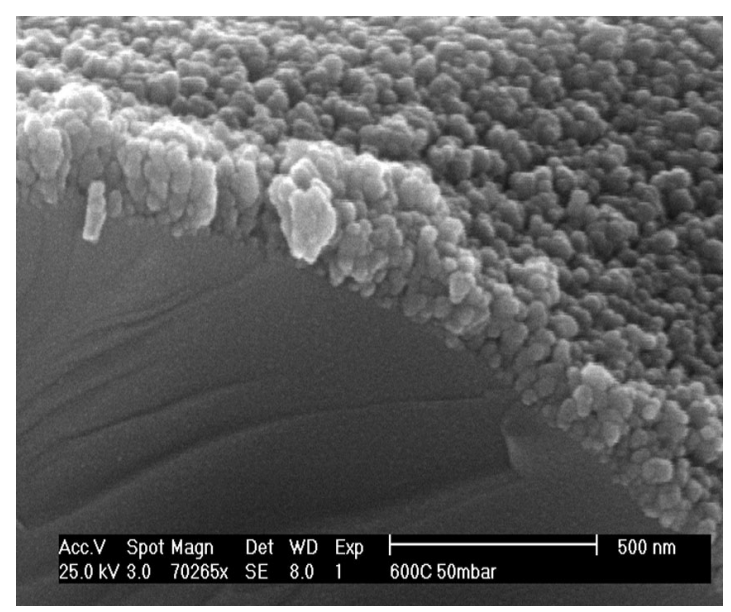

Figure 5: High resolution SEM of a nanocrystalline coating of yttrium stabilized zirconia on a porous anode substrate.

sive overview of the modeling is given by Winterer. [18] Recently, with the availability of large scale supercomputers and parallel PC clusters, the simulation of atomic processes involving millions of atoms has become available. Several authors have employed the molecular dynamics (MD) simulation technique to obtain details of the processes during particle formation, agglomeration, and sintering. As the nanoparticles contain only a limited number of atoms, it is possible to study diffusion and rearrangement processes leading to aggregation and particle growth. Atomistic simulations are extremely useful in describing the initial stages of sintering and equilibrium particle morphologies which determine the final structure and properties of 
nanoparticles prepared in the gas phase. Figure 6 shows the time evolution of Ge-nanoparticles, the aggregation and sintering during condensation in an Ar-gas. [19] In order to be able to simulate this process, high concentrations of atoms in the gas phase have to be employed in order to be able to adapt the time scale to the computing capabilities. A scaling law was established which allows the comparison to experimental conditions. MD-simulations have been employed for many other processes in nanocrystalline materials such as the calculation of the elastic properties [20] of nanocrystalline nickel and the ,plastic and superplastic deformation of nanocrystalline metals [21-23] and the atomistic structure and energies of grain boundaries in nanocrystalline metals.

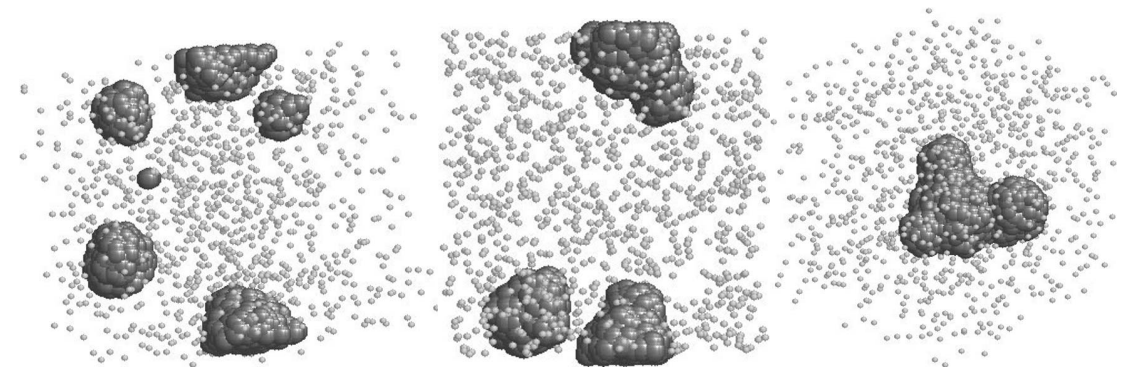

Figure 6: Result of a Molecular Dynamics (MD) simulation showing the evolution of Ge-nanoparticles in Argas for $0,96 \mathrm{ps} \mathrm{a}), 1,29 \mathrm{ps}$ b) and $3.36 \mathrm{~ns}$ ). The aggregation, sintering and change of morphology can be observed.

The EDOC process was developed to synthesize nanocrystalline oxides with improved properties as compared to oxides obtained by precipitation and sol-gel routes. $[11,24]$ In particular, it was attempted to improve the control on size and size distribution, the dispersibility and the possibility to modify the surfaces of the nanocrystalline powders. In the EDOC process an anode, i.e., metallic $\mathrm{Zn}$, is dissolved in an organic electrolyte with an organic conducting salt by applying a voltage. The $\mathrm{Zn}^{2+}$-ions migrate to an inert cathode, are discharged and subsequently oxidized by means of bubbling air through the electrolyte, thus forming ZnO-nanoparticles. The conducting salt has the additional role to prevent continued growth of the nanoparticles and their agglomeration. After filtration, the nanoparticles can be further modified or functionalized by exchanging the organic shell by other molecules to adapt the surface chemistry to other chemical environments. Other metal oxides have been prepared by EDOC, such as $\mathrm{SnO}_{2}, \mathrm{TiO}_{2}$, and $\mathrm{ZrO}_{2}$ as well as doped oxides. Compared to other wet chemical processes, the EDOC nanopowders exhibit superior dispersibility in solvents.

In conclusion, it can be stated that many synthesis techniques exist with individual advantages and disadvantages depending on the requirements of the material. In many processes, the microstructure, the morphology, the size and size distribution, the agglomeration and the elemental distribution on the scale of the nanoparticles can be controlled. In addition, it is possible to control the surface chemistry and thus to control the reactivity of the nanomaterials with the environment. The availability of versatile synthesis techniques is the prerequisite for materials design on the nanometer scale. 


\section{Characterization}

The characterization of nanostructured materials in the form of thin films, nanoparticles and bulk structures demands special techniques which allow for the structural and chemical analysis with a sufficient lateral resolution. Consequently, special characterization techniques are required besides the standard techniques available to materials science. The extremely high surface and interface areas have to be considered. Experimental difficulties such as oxidation/reaction at surfaces, the disordered structure at the grain boundaries and other internal interfaces and substantial porosity can be present in these materials. The specific surface area of nanopowders with average grain sizes below $10 \mathrm{~nm}$ can rise to several hundred $\mathrm{m}^{2} / \mathrm{g}$. That is, two to three grams of nanopowders which fit into a small volume of a few $\mathrm{cm}^{3}$ can have the same surface area as an entire football field. Therefore, ,nanocrystalline powders are considered to be excellent candidates for catalysis and gas sensing devices.

The crystallinity even in materials with average particle/grain sizes below $10 \mathrm{~nm}$ is very good. Figure 7 shows an X-,,ray diffractogram of nanocrystalline zinc oxide together with the Rietveld fit. An average particle size of $8 \mathrm{~nm}$ was determined independently by HRTEM and from the fit of the line broadening. The clear presence of all crystalline maxima indicates an excellent crystallinity of the nanopowders after the preparation and without any calcination treatment. The crystallinity of nanoparticles from the CVS process is even better which can be observed clearly in the high resolution TEM shown in Figure 8 for the case of zirconia.

An important aspect is the analysis of the elemental distribution on the scale of the nanoparticles. Only a few techniques provide the information with sufficient lateral resolution. HRTEM in combination with an Omega filter is a new technical solution to increase the analytical capabilities of transmission electron microscopy. Winterer describes the possibility to use EXAFS in

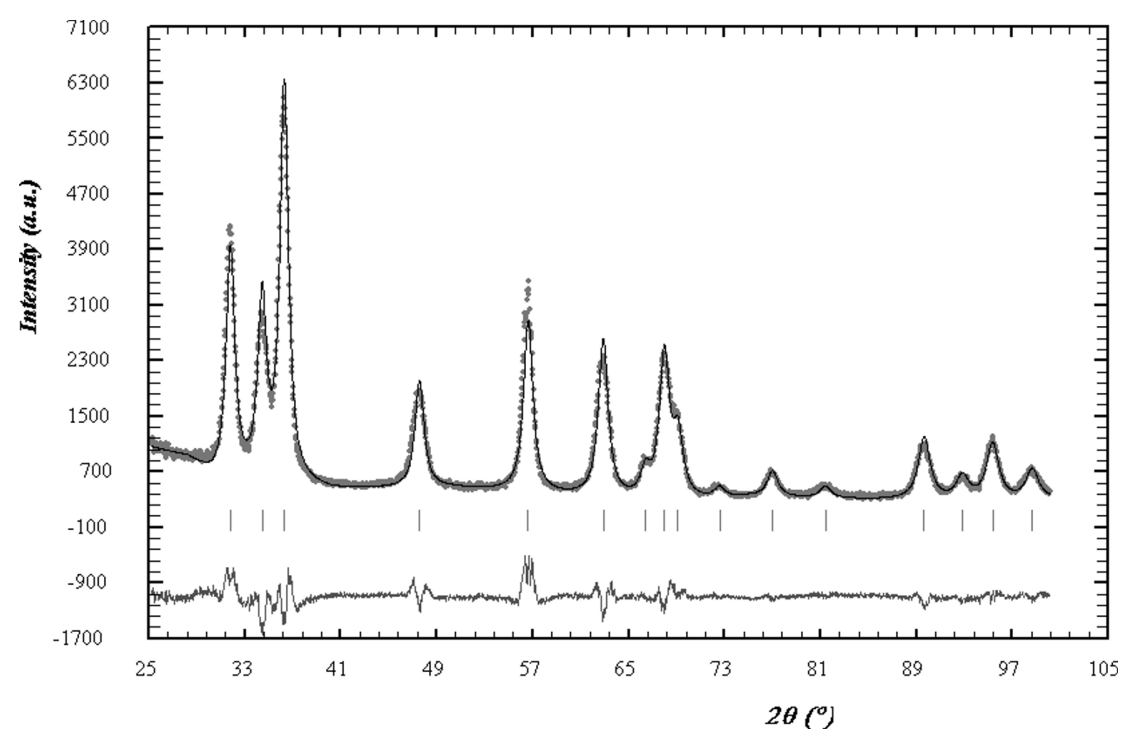

Figure 7: X-ray diffractogram of nanocrystalline $\mathrm{ZnO}$ powder prepared by EDOC. The experimental data, the Rietveld fit and the difference plot are shown. 


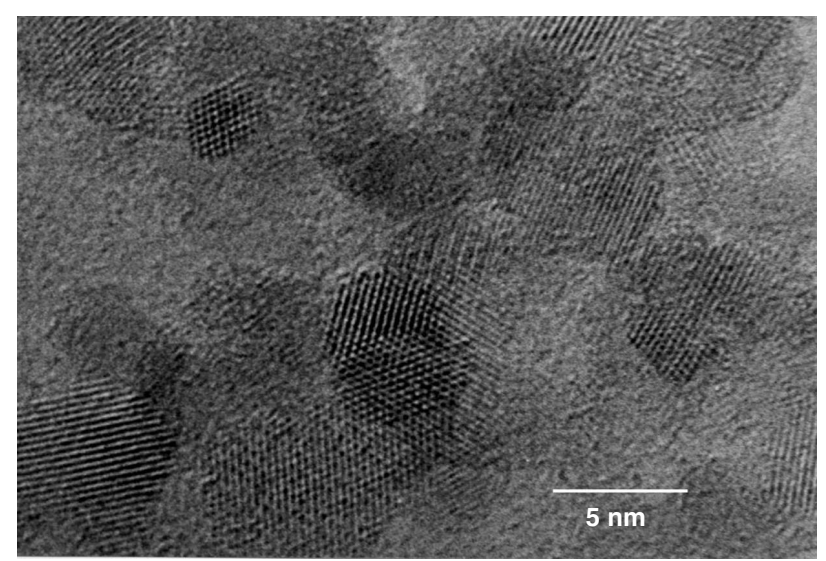

Figure 8: High Resolution TEM of as prepared CVS-zirconia nanoparticles. The lattice fringes extend to the surface proving excellent crystallinity. A narrow size distribution and crystallographic habitus planes at the surfaces are observed.

combination with Reverse Monte Carlo simulation to obtain the detailed structure and elemental distribution in nanometer sized particles. [25] By analyzing the pair distribution functions of $\mathrm{Zr}-\mathrm{Zr}, \mathrm{Zr}-\mathrm{Y}$, and $\mathrm{Y}-\mathrm{Y}$ it was shown that depending on the synthesis parameters a surface layer rich in yttrium oxide is formed instead of the expected yttrium doped zirconia. Other standard characterization techniques were not capable to resolve the thin surface layer with a different composition. It should be pointed out that a change of the synthesis conditions resulted in a homogeneously doped oxide. The knowledge of the detailed elemental distribution is extremely important in order to establish the structure-property relationships and to understand the complex behavior of nanomaterials. Other frequently used characterization techniques include nitrogen adsorption for porosity and pore size distribution, [26] small angle neutron scattering (SANS) for particle and pore size determination, [27] surface analytical techniques such as Xray photoelectron spectroscopy (XPS), ion scattering spectroscopy (ISS), nuclear techniques such as Mössbauer spectroscopy and many more depending on the details of interest.-

\section{$5 \quad$ Properties}

The interest in nanostructured materials arises from the fact that due to the small size of the building blocks and the high density of interfaces (surfaces, grain and phase boundaries) and other defects such as pores, new physical and chemical effects are expected or known properties can be improved substantially. In addition, the novel processing routes allow a bottom-up approach in materials design. In the ,following sections several examples of properties which are altered dramatically in the nanometer regime are presented.- 


\subsection{Nanoparticles}

The main reason for using nanoparticles is the large surface area which will be favorable for gas-solid interactions such as in catalysis [28] and gas sensing. [29] In addition, nanoparticles are the building blocks of bulk nanocrystalline materials, i.e., ceramics, prepared by sintering. Several authors have examined the catalytic activity of nanocrystalline materials. In catalysis, alumina with a high specific surface area is used extensively as support material for catalytically active noble metals. However, the role of the substrate material was not studied extensively. For several systems it was found that the influence of the substrate material on the catalytic performance in gas reactions, such as complete oxidation of methane, can be quite drastic. [30-32] Another application of the surface reactivity of nanocrystalline oxides is in gas sensing devices. As an example, nanocrystalline titania prepared by CVS was used for gas sensing. [29] A typical experimental arrangement consists of an alumina substrate with an interdigitized finger-like metallic structure on one side and a resistive heater on the other side. This allows the deposition of the sensing material and the measurement of the resistivity as a function of the atmosphere. As shown in Figure 9 a nanocrystalline coating was obtained by screen printing a dispersion and a moderate sintering step at $600{ }^{\circ} \mathrm{C}$ for one hour. The film is highly porous which allows the reactive gases to reach all surfaces of the sensing material in short times. All grains are in good contact to each other and sintering necks with well developed grain boundaries are formed. This

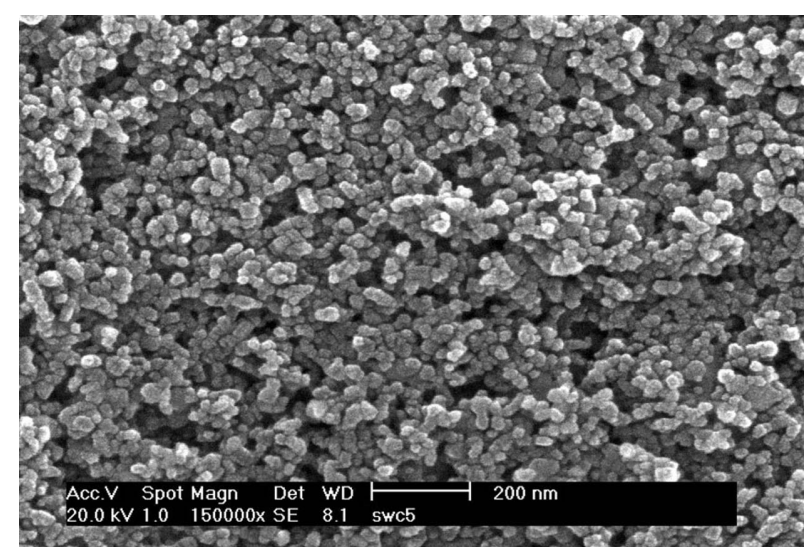

Figure 9: High resolution SEM demonstrating the nanocrystalline structure with nanoporosity of the titania layer prepared by screen printing and sintering.

is an important prerequisite for the measurement of the resistance of the film. As seen in Figure 10 , the reaction to a change of atmosphere, i.e. exposure to a different concentration of oxygen, is extremely fast, with response times (between 10 and $90 \%$ of the total signal) in the order of less than three seconds. This compares to the response times of several minutes for commercial sensors which are based on dense thin films deposited by physical vapor deposition such as sputtering. The fast response time is an attractive feature of the nanostructured gas sensors and is a direct consequence of the nanocrystalline and nanoporous structure. 


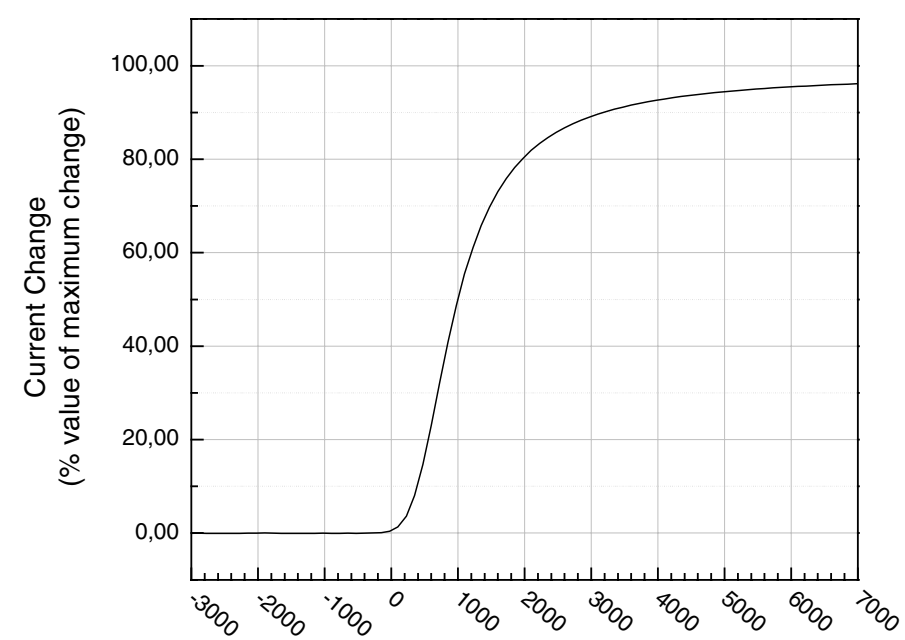

Figure 10: Change of the signal of a sensor made of nanocrystalline titania in response to a change of the oxygen concentration in the atmosphere. The sensor temperature was set at $300{ }^{\circ} \mathrm{C}$. The reaction time of the sensor, i.e. the time between $10 \%$ and $90 \%$ of the final signal is less than $3 \mathrm{~s}$.

Other interesting physical effects are observed in metallic nanoparticles such as FePt prepared by a wet chemical process described by Sun et al. [33] Fe50Pt50 nanoparticles in the size range from three to seven nanometers can be prepared with an organic coating of oleic acid which prevents the oxidation even when exposing the nanoparticles to air. [34] The ,organic shell also serves to keep the nanoparticles at a molecular distance. Therefore, the nanoparticles are superparamagnetic and can be arranged on clean surfaces in regular arrays which opens interesting applications for magnetic storage ,devices.

\subsection{Nanoceramics}

Nanoceramics with grain sizes in the range of 10 to $100 \mathrm{~nm}$ are typically prepared by consolidation, i.e., uniaxial compaction, cold isostatic pressing and sintering in air or vacuum. The green density, i.e. the density after initial compaction, reaches values of 40 to $50 \%$ of the theoretical value for CVS nanopowders. The most striking feature in the case of CVS powders is the transparency of the compacted pellet. This is a direct consequence of the narrow size distribution of pores in the material which can only occur if no agglomerates are present in the as prepared powder or are broken up during the consolidation step. The dependence of the green density with increasing compaction pressure does not show any change in slope which would be an indication of breakage of agglomerates. The size distribution shows only pores smaller than the primary particle size. This is further evidence for the lack of agglomeration in CVS powders. This feature is a prerequisite for a good sinterability of the CVS powders which has been found to occur in all nanoceramics under investigation $\left(\mathrm{TiO}_{2}, \mathrm{ZrO} 2, \mathrm{Y}_{2} \mathrm{O}_{3}, \mathrm{Al}_{2} \mathrm{O}_{3}\right.$, various doped oxides etc.) at temperatures well below $1 / 2 \mathrm{~T}_{\mathrm{M}}$. In the intermediate sintering steps at lower temperatures, the pores disappear but no excessive pore growth is observed. However, in pure 
ceramics grain growth occurs when the density exceeds $90 \%$ of the theoretical value. This undesirable effect has been reduced by doping, surface doping (core-shell nanoparticles) and composite structures. [13,14] In Figure 11 the grain size is plotted as a function of the sintering temperature for several oxides.

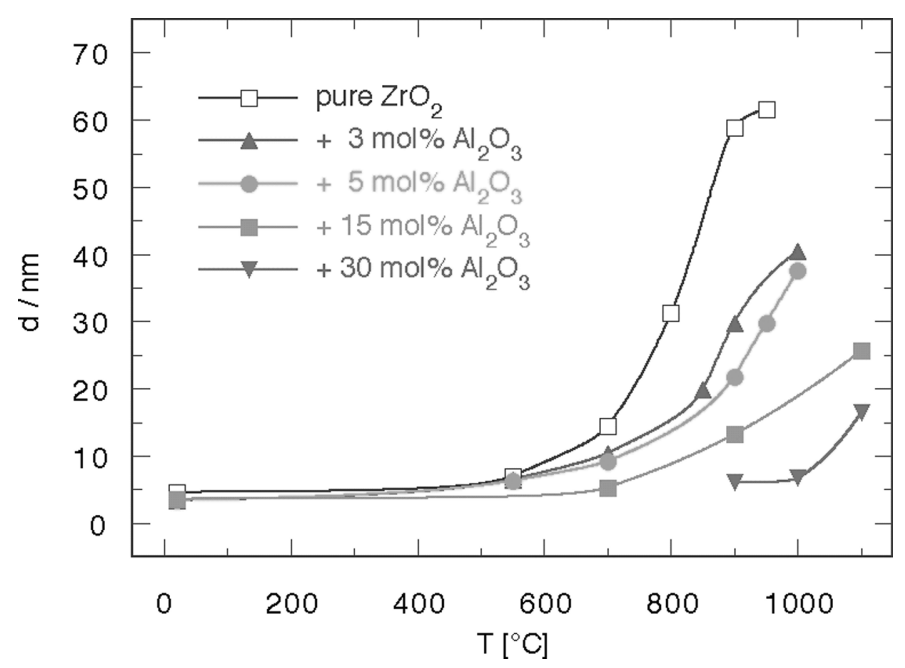

Figure 11: Grain size as a function of sintering temperature for pure zirconia and zirconia doped with four different concentrations of alumina. Only the $\mathrm{ZrO}_{2} / 30$ mol.- $\% \mathrm{Al}_{2} \mathrm{O}_{3}$ nanopowder is amorphous in the as prepared state, all other samples are crystalline with the tetragonal structure. The crystallization of the amorphous powder occurs at a temperature of approx. $800{ }^{\circ} \mathrm{C}$. Pure $\mathrm{ZrO}_{2}$ shows excessive grain growth with an average grain size typically exceeding $100 \mathrm{~nm}$, while the grain growth is effectively reduced by the doping.

In the pure nanocrystalline zirconia extensive grain growth is observed at temperatures and in the fully dense ceramic (100\% TD) sintered at $1000^{\circ} \mathrm{C}$ the average grain size is typically above $100 \mathrm{~nm}$. Fully dense ceramics are also obtained for zirconia doped with 3 to $5 \mathrm{~mol} \%$ alumina at grain sizes in the range of 40 to $50 \mathrm{~nm}$. Further increase of the doping to $15 \mathrm{~mol} \%$ further reduces the grain growth; however at a sintering temperature of $1000{ }^{\circ} \mathrm{C}$ the theoretical density is not reached. At a higher doping level of 30 mol.-\% alumina, the as prepared nanopowders are amorphous, crystallize at the sintering temperatures above $600{ }^{\circ} \mathrm{C}$ and show a phase separation. At this doping level the grain growth is suppressed most effectively as the grains grow only up to $30 \mathrm{~nm}$ even at $1200^{\circ} \mathrm{C}$. It should be mentioned that the crystallographic structure of as prepared pure zirconia with average grain size below $10 \mathrm{~nm}$ is monoclinic and tetragonal, while in the alumina doped samples the tetragonal structure is stabilized. Core-shell particles with an alumina rich surface and two phase zirconia-alumina structures prepared with the CVS method as described above also exhibit a drastic effect on the grain boundary mobility. In summary of the study of various zirconia ceramics, it can be stated that during pressureless sintering 1) pure nanocrystalline oxides exhibit extensive grain growth at high densities; 2) grain growth can be suppressed effectively by doping with different elemental distribution of the doping element; 3 ) depending on the concentration of the doping element fully dense and nanoporous, nanocrystalline ceramics can be obtained which are stable against pore and grain growth at temperatures exceeding $1000{ }^{\circ} \mathrm{C}$. The latter ceramics could be of interest for high temperature gas filters and 
as catalyst support materials at high temperatures for gas phase reactions. The densification can be further enhanced by the application of a high uniaxial or hydrostatic pressure while grain growth is further suppressed.

The grain size can influence the properties of nanomaterials in various ways. As shown for sintering the basic processes leading to densification and grain growth are identical and consequently, the properties are enhanced according to the grain size dependence. However, it is also possible that new processes lead to a different dependence on grain size. As an example, the plastic or superplastic deformation of metals and ceramics with nanocrystalline grain sizes is described. In metallic systems the Hall-Petch relationship describes the grain size dependence as a result of the interaction of dislocations and grain boundaries. Several theoretical approaches lead to a grain size dependence of the hardness or mechanical strength. The hardness increases as the inverse of the square root of the grain size. Several authors have reported that this well established behavior changes drastically as the grain size gets below a critical value in the nanometer regime. In this case the material becomes softer as the grain size is reduced and consequently, the effect is named Inverse Hall-Petch effect. It is obvious that different processes which require a lower stress than the dislocation-grain boundary interaction are operative in the nanometer regime. Grain boundary sliding is an alternative process, however, the steric hindrance by neighboring grains has to be overcome. Many theories describe dislocation and diffusion processes which relax the high stresses at the triple junctions when sliding occurs between grains. An alternative process based on the formation of mesoscopic grain boundaries has been proposed for nanocrystalline materials. [35] By grain boundary migration the grain morphology is altered to produce a flat mesoscopic interface over the dimensions of many grain diameters. The threshold stress for the smoothening of the grain boundaries has been calculated and found to decrease with decreasing grain size, i.e., an opposite behavior as in materials with larger grain sizes. In the model the basic step of the sliding is described by local atomic rearrangements of grain boundary atoms at the stress concentration sites leading to the sliding of the neighboring grains. As dislocations in the grains do not play a role in this process mesoscopic sliding can be active in both metals and ceramics. The model of a new process responsible for the deformation of nanocrystalline metals and ceramics has been further supported by molecular dynamics simulations of nanocrystalline nickel at different grain sizes. [36] It is found that at the smallest grain sizes the dominant processes occur in the interfaces leading to grain boundary sliding, atomic shuffling in the interfaces, grain rotation etc. However, in these materials no dislocation activity is observed in agreement with the model predictions. At larger grain sizes, the effects of dislocation activity are observed as stacking faults indicate the motion of dislocations through the grains. However, MD requires very short time scales in the range of nanoseconds and therefore, high stresses which do not correspond to experimental values are applied. The consequences for the atomic processes are not clear. In summary, it can be stated that for nanocrystalline materials a transition from dislocation based mechanisms in larger grains to an interface controlled sliding mechanism seems most likely as it explains the observed change of grain size dependence. The combination of deformation experiments, theoretical models and atomistic simulations provide an insight into the complex deformation processes of nanocrystalline materials. 


\section{Applications}

Nanotechnology is already used extensively in modern industrial products. The semiconductor industry which relies on the miniaturization of the structural components has used device structures in the nanometer range in commercial products. The time to market in this industry is extremely short as the demand by the end user is very high. An example for the fast realization of a technical product based on a new physical effect is the giant magneto resistance (GMR) effect originally discovered in 1985. A few years later the first hard disks with a GMR-based read head were in the market and a revolutionary increase in storage density leading to smaller hard disks followed.

Over decades several companies have marketed agglomerated powders in many industrial applications. Although the primary particle size is in the nanometer range, properties such as dispersibility, light scattering and sinterability are determined by the size of the agglomerates or aggregates. The continued need for better products can only be fulfilled by improved materials and consequently, many companies have intensified research in this area. In developing new products companies have to consider the costs of new materials and technologies as private and industrial customers are not willing to \(buy nano, but only improved products and processes\). For the continuing support of basic science in the field of nanomaterials and nanotechnology it is of utmost importance that the new properties and technologies also lead to commercial successes. The range of ideas for commercial products in nanomaterials is as wide as the field of materials science, covering mechanical, physical, chemical, biological, pharmaceutical, medical and cosmetic areas. Several companies have introduced into the market sun screen products based on $\mathrm{TiO}_{2}$ and $\mathrm{ZnO}$ nanopowders in an attempt to reduce the amount of organic sun blockers at the same sun protection factor. An important consideration for many applications is the dispersibility of the nanopowders in various liquid (dispersions or slurries for ceramic processing) and solid media (nanocomposites) which requires surface modifications to adapt the inorganic surface to the matrix. Ferrofluids, i.e., magnetic fluids, which have been in commercial use for many years are an example of the extremely good stability of liquid dispersions despite the strong magnetic interaction forces. Dispersions with excellent stability have been synthesized and are used in many processes. The use of nanopowders in semiconductor processing for chemical mechanical polishing (CMP) is a huge market and an important component in the manufacturing of modern integrated circuits with three-dimensional (3D) architecture. In the field of catalysis and gas sensors nanocrystalline powders and porous ceramics are considered. The development of new products by transferring basic science results has led to the foundation of numerous start-up companies as well as the involvement of large international companies in many countries. Some examples in the area of nanomaterials are Nanophase Technologies, Nanopowder Enterprises in the United States, Samsung Corning in Korea, APT in Australia, SusTech Darmstadt, NanoGate, and Degussa in Germany. It is anticipated that in the next few years many approaches will lead to the use of nanomaterials and only future can tell which attempt will also be a commercial success. 


\section{$7 \quad$ Acknowledgement}

The author acknowledges the financial support of the research work by the Deutsche Forschungsgemeinschaft, the Bundesministerium für Forschung und Technologie, the Alexandervon-Humboldt Stiftung, the Deutsche Akademische Austauschdienst, the Hahn-Meitner-Institut Berlin and SusTech GmbH \& Co. KG Darmstadt.

The plentiful contributions and discussions by PD Dr. habil. M. Winterer, Dr. B. Stahl, Prof. K. Albe, Dr. M. Ghafari, Prof. A. Raju, Dr. A. G. Balogh, Dipl.-Ing. J. Seydel, M. Sc. YongSang Cho and other members of the Thin Films Division, and Dr. W. Miehe and Prof. H. Fuess of the Structural Research Division in the Institute of Materials Science, TU Darmstadt, and Prof. V. Srdic, Novi Sad, Serbia and Montenegro, Prof. K. A. Padmanabhan, Hyderabad, India, Prof. G. Hoflund, Gainesville (USA) are appreciated.

\section{References}

[1] R. Z. Wang, Z. Suo, A. G. Evans, N. Yao, I. A. Aksay, J.'Mater. Res. 2001, 162485.

[2] W. Barthlott, C. Neinhuis, Planta 1997, 202, 1

[3] I. A. Aksay, M. Trau, S. Manne, I. Honma, N. Yao, L.'Zhou, P. Fenter, P. M. Eisenberger, S. M. Gruner, Science 1996, 273, 892

[4] S. Busch, H. Dolhaine, A. DuChesne, S. Heinz, O. Hoch,,rein, F. Laeri, O. Podebrad, U. Vietze, Th. Weiland, R.' Kniep, Eur. J. Inorg. Chem. 1999, 1643

[5] For some examples in magnetic nanocomposites, nanodispersions, and others, see www.sustech.de

[6] H. Gleiter, Proc. of the 7th Riso Int. Symposium on Metallurgy and Materials Science (1981), Roskilde, pp. 15-21

[7] R. Smalley, R. Curl, H. Kroto, Nobel Prize in Chemistry 1996

[8] Heinrich Rohrer, Gerd Binnig, Nobel Prize in Physics 1985

[9] A. M. El-Sherik, U. Erb, J. Mater. Sci. 1995, 30, 5743

[10] W. Chang, G. Skandan, H. Hahn, S. C. Danforth, B. H. Kear, Nanostructured Mater. 1994, 4, 345

[11] A. Dierstein, H. Natter, F. Meyer, H.-O. Stephan, C.'Kropf, R. Hempelmann, Scripta Mat. 2001, 44, 2209

[12] Degussa, Hanau, http://www.degussa.com

[13] V. V. Srdic, M. Winterer, H. Hahn, J. Am. Ceram. Soc. 2000, 83, 1853

[14] V. V. Srdic, M. Winterer, A. Möller, G. Miehe, H. Hahn, J. Am. Cer. Soc. 2001, 84, 2771

[15] I. Lamparth, D. Szabo, D. Vollath, Macromol. Symp. 2002, 181, 107

[16] M. Schallehn, M. Winterer, T. Weirich, U. Keiderling, H.'Hahn, Chem. Vap. Dep. 2003, 9,40

[17] J. Seydel, M. Winterer, H. Hahn, Mat. Res. Soc. Symp. Proc 2001, 676, Y8.14.1-5

[18] M. Winterer, Nanocrystalline Ceramics, Synthesis and Structure, Springer Series in Materials Science 53, New York 2002

[19] P. Krasnochtchekov, K. Albe, Y. Ashkenazy, R. S. Averback, J. Phys. Chem., submitted

[20] S.-J. Zhao, K. Albe, H. Hahn, Acta Mater., submitted 
[21] H. Van Swygenhoven, D. Farkas, A. Caro, Phys. Rev. B 2000, 62, 831

[22] V. Yamakov, D. Wolf, M. Salazar, S. R. Phillpot, H. Gleiter, Acta. Mater. 2001, 49, 2713

[23] K. W. Jakobsen, J. Schiotz, Nature Materials 2002, 1, 115

[24] R. Hempelmann, H. Natter, EP 1121 477, 2003

[25] M. Winterer, J. Appl. Phys. 2000, 88, 5635

[26] H. Hahn, Nanostructured Mater. 1993, 2, 251

[27] U. Keiderling, A. Wiedenmann, V. Srdic, M. Winterer, H. Hahn, J. Appl. Cryst. 2000, 33, 483

[28] H. Hahn, H. Hesemann, W. Epling, G. B. Hoflund, Mat. Res. Soc. Symp. 1998, 497, 35

[29] Yong-Sang Cho, Ph.D. Thesis, Technische Universität Darmstadt 2003

[30] G. B. Hoflund, Z. Li, W. S. Epling, T. Göbel, P. Schneider, H. Hahn, React. Kinet. Catal. Lett. 2000, 70, 97

[31] A. Tschöpe, D. Schaadt, R. Birringer, J. Y. Ying, Nanostructured Mater. 1997, 9, 423

[32] S. H. Oh, M. L. Everett, G. B. Hoflund, J. Seydel, H. Hahn, Mat. Res. Soc. Symp. Proc. 2001, Y4.6.1-6, 676

[33] S. Sun, C. B. Murray, D. Weller, L. Folks, A. Moser, Science 2000, 287, 1989

[34] B. Stahl, N. S. Gajbhiye, G. Wilde, D. Kramer, J. Ellrich, M. Ghafari, H. Hahn, H. Gleiter, J. Weißmüller, R.'Würschum, P. Schlossmacher, Adv. Mater. 2002, 14, 24

[35] H. Hahn, K. A. Padmanabhan, Phil. Mag. B 1997, 76, 559

[36] A. Hasnaoui, H. Van Swygenhoven, P. M. Derlet, Phys. Rev. B 2002, 66, 184112 